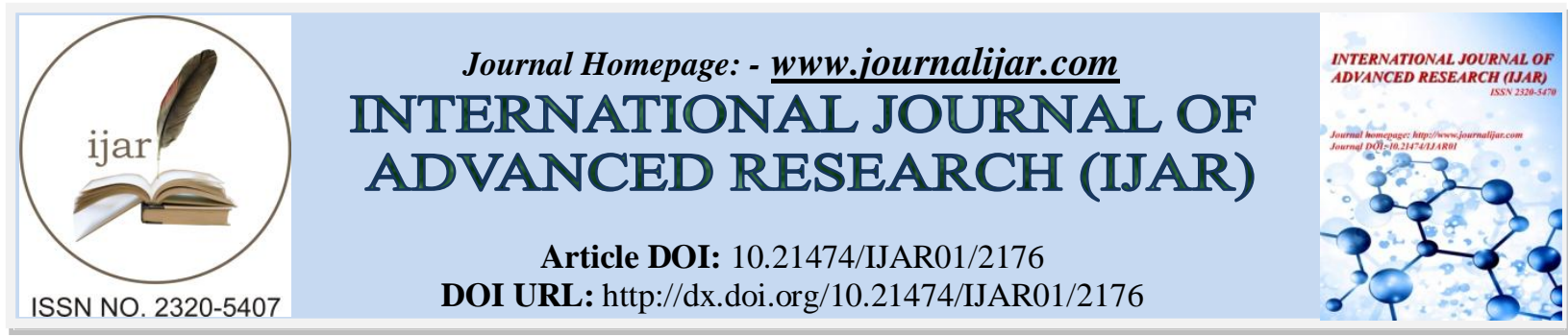

RESEARCH ARTICLE

\title{
RECURRENT ACUTE HIDRADENITIS SUPPURATIVA: CASE REPORT WITH 12 MONTH FOLLOW
} UP.

Dr. Abrar Abdullah Alsharif.

medical intern, Om Alqura University, Saudi Arabia.

\section{Manuscript Info}

(.........................

Manuscript History

Received: 26 September 2016

Final Accepted: 29 October 2016

Published: November 2016

\section{Abstract}

Hidradenitis suppurativa usually occurs in otherwise healthy adolescents and adults. It rarely may begin before puberty. In a significant number of cases, no explanation exists. This case report documents one patient with a clear pattern of recurrent acute hidradenitis suppurativa and no identifiable cause despite great effort. After 8 months of recurrent symptoms, she was found to have marked elevation of white blood cell and erythrocyte sedimentation rate. The onset of hidradenitis suppurativa is insidious, with the earliest sign being erythema and may present with variable clinical features. Therefore, it is important to approach hidradenitis suppurativa as a systemic disease with an interprofessional team.

Copy Right, IJAR, 2016,. All rights reserved.

\section{Presenting Concerns:-}

The subject of this report is a 45 year old male patient known case of diabetic mellitus come throw emergency department complaining of multiple painful swelling in left groin associated with purulent discharge. The swelling started small size and after 3 day increase in size with more painful. No history of fever or arthritis. He is smoker 2 packet daily since 20 year. He is a previous history in bilateral axilla before 2 years and treatment by surgery. The systemic review unremarkable. Past medical history include diabetes mellitus type 2 on metformin $500 \mathrm{mg}$ TID and aspirin $85 \mathrm{mg}$ once daily. Past surgical history are incision and drainage bilateral axillary abscess before 2 years and appendectomy before 6 years. No family history in the same condition. He is married, Soldier work and live in makkah.

\section{Clinical Findings:-}

The patient noted small multiple swelling in left groin side and one swelling in scrotum and after 3 day the swelling increase in size with more painful and some pus discharge . when examine the patient he was conscious, oriented. Her temperature was 37.2 , blood pressure was $115 / 79$, pulse $74, \mathrm{Po}_{2} 99 \%$ and $\mathrm{BMI} 28,7 \mathrm{~kg} / \mathrm{m} 2$. Her physical exam was unremarkable. Local examination include multiple swelling in left groin about $2 \times 1.5 \mathrm{~cm}$ and swelling in left scrotum about $1 \times 1.5 \mathrm{~cm}$ and Multiple open comedones with purulent discharge but more in groin side with erythema, hotness and tenderness.

The diagnosis is primarily clinical, no pathognomonic test exists, and biopsy is rarely required, especially in welldeveloped lesions. The consensus approach indicates that three key elements are required to diagnose hidradenitis suppurativa: typical lesions, characteristic 
distribution, and recurrence. Arbitrarily, two recurrences over a period of 6 months have been used as a qualifier for a diagnosis. All three criteria must be present for the definitive diagnosis. Hidradenitis suppurativa has a predilection for the intertriginous regions. The axillary and inguinoperineal regions are most commonly affected. In this patient Multiple open comedones and so-called bridged comedones are the hallmark finding of hidradenitis suppurativa; they frequently progress to multiple abscesses and sinus tract formations ${ }^{[1]}$. When 2 distant cutaneous orifices are interconnected through a subcutaneous fistula, they form bridging lesions. As the disease becomes chronic, large scars and contractures develop with persistent erythema. Inguinal-anogenital infiltration involves brown-red lesions with pus, blood, and a foul-smelling secretion that emerges from the numerous fistular openings ${ }^{[2]}$.

\section{Diagnostic Focus And Assessment:-}

The differential diagnosis for recurrent hidradenitis suppurativa is well established (Table 1) ${ }^{[3]}$. Her evaluation demonstrated no structural or anatomic explanations as well as no classic metabolic and autoimmune etiologies. Although her workup had met or exceeded all published guidelines, she had no explanation and no plan for preventing future episodes.

Table[1]:- Differential diagnosis of Hidradenitis Suppurativa

\begin{tabular}{|l|}
\hline Blastomycosis \\
\hline Catscratch disease \\
\hline Dermatologic aspect of acnimycosis \\
\hline Dermatologic manifestation of lymphogranuloma venerum \\
\hline Dermatologic manifestation of necardiosis \\
\hline Erysipelas \\
\hline Granuloma inguinale \\
\hline Noduloulcerative syphilis \\
\hline
\end{tabular}

The laboratory tests includes: CBC count erythrocyte sedimentation rate, C-reactive protein, Urinalysis and serum protein electrophoresis. All of this laboratory in the normal range except elevated erythrocyte sedimentation rate, elevated white blood cell count and serum protein abnormalities on electrophoresis. Ultrasonography of the hair follicles and dermal thickness of groin region in this patients are abnormalities in the deep part of the follicle. Thickened skin may play a role in the pathogenesis of hidradenitis suppurativa. Clinical staging of hidradenitis suppurativa has diagnostic value. A study has shown that the soluble IL-2 receptor serum level in patients with hidradenitis suppurativa can be used as valuable marker for disease staging. It is as follows:

- First stage: Solitary/multiple, isolated abscess formation without scarring or sinus tracts

- Second stage: Recurrent abscesses, single/multiple widely separated lesions, with sinus tract formation and cicatrization. Third stage: Diffuse/broad involvement or multiple interconnected sinus tracts/abscesses across the entire area. Dynamic staging system have been used for assessing differences in treatment effects and including the following: ${ }^{[4]}$

- Anatomical region involved (axilla, groin, genital, gluteal, or other inflammatory region left and/or right) Three points per region involved

- Number and scores of lesions (abscesses, nodules, fistulas, scars) - Two points for each nodule, 4 points for each fistulas, 1 point for each scar, 1 point for each "other"

- The longest distance between 2 relevant lesions, ie, nodules and fistulas, in each region, or size if only 1 lesion Less than $5 \mathrm{~cm}, 2$ points; less than $10 \mathrm{~cm}, 4$ points; more than $10 \mathrm{~cm}, 8$ points

- Lesions are clearly separated by normal skin in each region - If yes, 0 points; if no, 6 points ${ }^{[3]}$.

\section{Therapeutic Focus And Assessment:-}

Surgery is necessary at times, especially in chronic hidradenitis suppurativa. In this patient, wide surgical excision is needed Because the wound is deep, the area needs to be covered with a skin flap (skin from nearby is pulled over to cover the wound). HS does not return to the treated area, but it can develop nearby.

Antibiotics( sefurxime) was prescribed at $500 \mathrm{mg}$ by mouth twice a day for 10 days. This is often part of the treatment plan. These drugs can reduce inflammation, fight infection, prevent HS from worsening, and stop new breakouts.

Diabetes drug: Metformin was taken at $500 \mathrm{mg}$ orally twice daily and it has been approved to treat adult-onset diabetes . It may also help people who have HS and a condition called metabolic syndrome. 


\section{Prognosis:-}

The impression of a relentlessly progressive disorder may be explained by the finding that almost two thirds of patients acknowledged the existence of persistently painful boils that failed to heal. Possibly, new boils develop at an unchanged rate throughout the course of the disease, but some fail to subside in the usual manner and become chronic $^{[5]}$.

With rare exceptions, surgical intervention is sufficient to stop the disease.Shame, frustration, and despair may cause patients to delay radical surgical procedures.

No single treatment has shown overwhelmingly positive outcomes

Spontaneous resolution is rare.

The recurrence rate in patients treated with surgery varies considerably depending on the site affected; the highest rate is $50 \%$ in the submammary region. An overall recurrence rate of $2.5 \%$ have been estimated after wide surgical excision, with a median postoperative follow-up of 36 months.

\section{Patient Education:-}

Patients should be educated about the initial treatments, which include the following:

- $\quad$ Practicing proper hygiene

- Using soaps and antiseptic and antiperspirant agents

- Using warm compresses

- Wearing loose-fitting clothing

- Smoking cessation

\section{Follow-Up And Outcomes:-}

After 12 months of follow-up, there have been no further episodes of hidradenitis suppurativa.

In September of 2016, repeat CBC count and erythrocyte sedimentation rate demonstrated normalization of white blood cell, serum protein and erythrocyte sedimentation rate.

\section{Discussion:-}

Initial evaluation fails to detect the cause of recurrent acute pancreatitis in $10 \%$ to $30 \%$ of patients or about 2,000 to 8,000 patients per year in the Saudi Arabia alone. In these patients, more extensive evaluations are warranted including recurrent in less than year and deep skin.

Even then, as in this case, such evaluations may not provide an explanation in a small percentage of patients. At this time, no published guidelines exist for further evaluation of idiopathic recurrent hidradenitis suppurativa .

In this case report, the patient's recurrent acute hidradenitis suppurativa appears related to markedly elevated white blood cell, serum protein and erythrocyte sedimentation rate.

Detailing the clinical features of the disease, Brunsting highlighted its frequent association with acne. He noted that hidradenitis suppurativa, dissecting cellulitis of the scalp and the neck, and acne conglobata commonly occur in the same patient. Plewig and Kligman pointed out that hidradenitis suppurativa is a misnomer because of the lack of apocrine gland involvement, but they did not present a detailed explanation ${ }^{[6]}$. Eventually, hidradenitis suppurativa was accepted as an acneiform disorder that begins with follicular occlusion rather than an infection of the sweat glands. The disorder has significant socioeconomic effects as well. Jemec et al documented, in the Danish population, an average of 2.7 lost work days per year due to hidradenitis suppurativa (overall workdays lost was 7.5). ${ }^{[7]}$ The general self-reported level of health, which is well correlated with more objective parameters of morbidity, was also significantly worse among hidradenitis suppurativa patients than healthy control subjects. The mean Dermatology Life Quality Index (DLQI) score for hidradenitis suppurativa is higher than for previously studied skin diseases, indicating significant morbidity for those affected.

No one knows for sure what causes HS. Because it occurs after puberty, hormones likely play a role. The person's immune system also seems to play a role. It is possible that HS develops when the person's immune system overreacts. HS begins in the hair follicles (where hair grows out of the skin). Like everyday acne, HS forms when the hair follicles clog with bacteria and other substances. It is possible that HS develops in people who have an 
immune system that overreacts to the plugged hair follicle. Lifestyle also seems to play a role $^{[8]}$. It seems that smoking, being overweight, or taking lithium may trigger HS. These things do not cause HS. If a person is susceptible to getting HS, any of these could make HS appear for the first time or worsen existing HS. The condition may also begin to occur in patients of any age after puberty, but rarely before. Cases vary in severity, but all require some treatment and management. For mild cases, home remedies may be all that is needed. For severe like this patient forms however, daily medication is often prescribed and radical surgery may eventually be recommended ${ }^{[9]}$. Early diagnosis and treatment of HS is important because it can help manage symptoms and prevent new boils and lesions from forming ${ }^{[10]}$.

\section{Patient Consent:-}

The patient provided written permission for publication of this case report.

\section{References:-}

1. [Guideline] Zouboulis CC, Desai N, Emtestam L, Hunger RE, Ioannides D, Juhász I, et al. European S1 guideline for the treatment of hidradenitis suppurativa/acne inversa. J Eur Acad Dermatol Venereol. 2015 Apr. 29 (4):619-44.

2. Wang SC, Wang SC, Alavi A, Alhusayen R, Bashash M, Sibbald RG. Hidradenitis Suppurativa: A Frequently Missed Diagnosis, Part 2: Treatment Options. Adv Skin Wound Care. 2015 Aug. 28 (8):372-80; quiz 381-2.

3. van der Zee HH, Jemec GB. New insights into the diagnosis of hidradenitis suppurativa: Clinical presentations and phenotypes. J Am Acad Dermatol. 2015 Nov. 73 (5 Suppl 1):S23-6.

4. von der Werth JM, Jemec GB. Morbidity in patients with hidradenitis suppurativa. Br J Dermatol. 2001 Apr. 144(4):809-13.

5. Alikhan A, Lynch PJ, Eisen DB. Hidradenitis suppurativa: a comprehensive review. J Am Acad Dermatol. 2009 Apr. 60(4):539-61; quiz 562-3.

6. Iwasaki J, Marra DE, Fincher EF, Moy RL. Treatment of hidradenitis suppurativa with a nonablative radiofrequency device. Dermatol Surg. 2008 Jan. 34(1):114-7

7. Gulliver W, Zouboulis CC, Prens E, Jemec GB, Tzellos T. Evidence-based approach to the treatment of hidradenitis suppurativa/acne inversa, based on the European guidelines for hidradenitis suppurativa. Rev Endocr Metab Disord. 2016 Feb 1. 35(2 Pt 1):191-4.

8. Hughes R, Kelly G, Sweeny C, Lally A, Kirby B. The medical and laser management of hidradenitis suppurativa. Am J Clin Dermatol. 2015 Apr. 16 (2):111-23.

9. Ah-Weng A, Langtry JA, Velangi S, Evans CD, Douglas WS. Pyoderma gangrenosum associated with hidradenitis suppurativa. Clin Exp Dermatol. 2005 Nov. 30(6):669-71.

10. Kurzen H, Kurokawa I, Jemec GB, et al. What causes hidradenitis suppurativa?. Exp Dermatol. 2008 May. 17(5):455-6; discussion 457-72 